OPEN ACCESS

Edited by:

Arumugam R. Jayakumar, Miami VA Healthcare System,

United States

Reviewed by:

Masato Hasegawa

Tokyo Metropolitan Institute

of Medical Science, Japan

Cemil Kerimoglu,

German Center

for Neurodegeneratives, Helmholtz Association of German Research

Centers (HZ), Germany

*Correspondence:

Chiara F. Valor

chiara.valori@dzne.de

Specialty section: This article was submitted to Neurodegeneration, a section of the journal

Frontiers in Neuroscience

Received: 14 June 2021

Accepted: 31 July 2021

Published: 01 September 2021

Citation:

Valori CF and Neumann M (2021) Contribution of RNA/DNA Binding

Protein Dysfunction

in Oligodendrocytes

in the Pathogenesis of the

Amyotrophic Lateral

Sclerosis/Frontotemporal Lobar

Degeneration Spectrum Diseases.

Front. Neurosci. 15:724891. doi: 10.3389/fnins.2021.724891

\section{Contribution of RNA/DNA Binding Protein Dysfunction in Oligodendrocytes in the Pathogenesis of the Amyotrophic Lateral Sclerosis/Frontotemporal Lobar Degeneration Spectrum Diseases}

\author{
Chiara F. Valori ${ }^{1 *}$ and Manuela Neumann ${ }^{1,2}$
}

${ }^{1}$ Molecular Neuropathology of Neurodegenerative Diseases, German Center for Neurodegenerative Diseases, Tübingen, Germany, ${ }^{2}$ Department of Neuropathology, University Hospital of Tübingen, Tübingen, Germany

Amyotrophic lateral sclerosis (ALS) and frontotemporal lobar degeneration (FTLD) are two incurable neurodegenerative disorders, often considered as the extreme manifestations of a disease spectrum, as they share similar pathomechanisms. In support of this, pathological aggregation of the RNA/DNA binding proteins transactivation response element DNA-binding protein 43 (TDP-43) or fused in sarcoma (FUS) is the pathological hallmark found in neurons and glial cells of subsets of patients affected by either condition (i.e., ALS/FTLD-TDP-43 or ALS/FTLDFUS, respectively). Among glia, oligodendrocytes are the most abundant population, designated to ensheath the axons with myelin and to provide them with metabolic and trophic support. In this minireview, we recapitulate the neuropathological evidence for oligodendroglia impairment in ALS/FTLD. We then debate how TDP-43 and FUS target oligodendrocyte transcripts, thereby controlling their homeostatic abilities toward the axons. Finally, we discuss cellular and animal models aimed at investigating the functional consequences of manipulating TDP-43 and FUS in oligodendrocytes in vivo. Taken together, current data provide increasing evidence for an important role of TDP-43 and FUS-mediated oligodendroglia dysfunction in the pathogenesis of ALS/FTLD. Thus, targeting disrupted oligodendroglial functions may represent a new treatment approach for these conditions.

Keywords: oligodendrocytes, ALS, FTLD, TDP-43, FUS

\section{INTRODUCTION}

Amyotrophic lateral sclerosis (ALS) is the most frequent neuromuscular disease characterized by the predominant loss of first and second motor neurons in the brain and spinal cord, leading to muscle weakness, paralysis, and death generally within 1-5 years (Hardiman et al., 2017). Frontotemporal dementia (FTD) is the second most common cause of presenile 
dementia characterized by the predominant degeneration of the frontal and temporal lobes (i.e., frontotemporal lobar degeneration, FTLD), causing personality and behavioral changes as well as difficulties formulating and understanding language (Piguet and Kumfor, 2020). In addition to a recognized clinical overlap (Burrell et al., 2016), seminal discoveries in the last decades have provided compelling molecular evidence that ALS and FTLD are closely related conditions sharing overlapping pathogenesis with dysfunctions of RNA binding proteins emerging as key roles. Briefly, trans-activation response element DNA-binding protein 43 (TDP-43) was discovered in 2006 as a disease protein in the most common form of FTLD and in almost all ALS cases (Arai et al., 2006; Neumann et al., 2006), now renamed as FTLD_TDP and ALS-TDP. They include sporadic and genetic cases with mutations in GRN and C9orf72 as the most common defects in addition to other rarer genetic forms with mutations in TARDBP, TBK1, VCP, OPTN, and UBQLN2 (Pottier et al., 2016). This was followed in 2009 by the identification of mutations in fused in sarcoma (FUS) in $\sim 5 \%$ of familial ALS (ALS-FUS) (Kwiatkowski et al., 2009; Vance et al., 2009) and the subsequent recognition of FUS aggregation together with TAF15 and EWS as hallmark lesions in $~ 10 \%$ of sporadic FTLD cases (FTLD-FUS) (Munoz et al., 2009; Neumann et al., 2009b,c).

TDP-43 and FUS the acronyms improve readability are ubiquitously expressed DNA/RNA binding proteins with a physiologically predominant nuclear localization, involved in multiple steps of RNA metabolism, including transcription, splicing, transport, and stabilization (Ratti and Buratti, 2016; Buratti, 2021). The mechanisms underlying TDP-43/FUS aggregation and neurodegeneration are complex and currently not fully understood. However, given the intimate association between cytoplasmic accumulation of abnormal TDP-43 or FUS associated with their nuclear depletion in a disease state, the current consensus is that most likely a combination of complex disturbances due to loss of nuclear and cytoplasmic functions and gain of toxic functions through aggregates is involved, with abnormal messenger RNA particle formation and dynamics as well as nucleocytoplasmic transport defects emerging as crucial factors (Bowden and Dormann, 2016; Ederle and Dormann, 2017). Although most previous and current research to dissect the pathomechanisms is focused on neurons, it has to be noted that oligodendrocytes are also strongly affected by TDP-43 and FUS pathology in ALS/FTLD (Figure 1; Neumann et al., 2007; Geser et al., 2008; Mackenzie and Neumann, 2017). Given the fact that oligodendrocytes share a developmental origin with spinal cord motor neurons (Lu et al., 2002) and are, like neurons, heavily dependent on messenger RNA (mRNA) transport over long distances for local translation, it is tempting to speculate overlapping aggregation mechanisms and that oligodendroglial dysfunction might contribute to TDP-43 and FUS disease pathogenesis as discussed for other neurodegenerative diseases (Ettle et al., 2016; Mot et al., 2018).

Here, we review the neuropathological and genetic evidence of oligodendrocyte impairment in TDP-43/FUSproteinopathies, summarize current insights into the roles of TDP-43 and FUS in oligodendrocytes, and discuss the potential impact of altered oligodendroglial functions in ALS/FTLD pathogenesis.

\section{NEUROPATHOLOGICAL AND GENETIC EVIDENCE FOR OLIGODENDROGLIAL DYSFUNCTION IN HUMAN TRANS-ACTIVATION RESPONSE ELEMENT DNA-BINDING PROTEIN 43 AND FUSED IN SARCOMA/FET PROTEINOPATHIES}

Intracellular accumulation of hyperphosphorylated TDP-43 with loss of physiological nuclear TDP-43 is the hallmark in the most common form of frontotemporal lobar degeneration (FTLD - TDP) and in the vast majority of cases of ALS (ALSTDP). Neuronal TDP-43 pathology presents as compact or diffuse neuronal cytoplasmic inclusions, neuronal intranuclear inclusions, dystrophic neurites, and thread and dot pathology. Based on the morphology, amount, and distribution pattern of cortical neuronal inclusions, at least five distinct subtypes of FTLD-TDP pathology can be delineated (types A-E), each with relatively specific clinical and genetic associations and recognition that cortical pathology in ALS-TDP most closely resembles FTLD_TDP type B (Mackenzie and Neumann, 2017; Neumann et al., 2021). The presence of additional TDP-43 pathology in oligodendrocytes as glial cytoplasmic inclusions (GCIs) (Figure 1) was already described briefly after the initial discovery of TDP-43 as disease protein in FTLD-TDP (Neumann et al., 2007) and ALS-TDP (Mackenzie et al., 2007) but was only more recently studied in more detail. In fact, GCI pathology in the gray and white matter of the spinal cord, precentral gyrus, and middle frontal gyrus is reported as a highly characteristic feature of almost all ALS-TDP cases, including sporadic and genetic forms with mutations in C9orf72, TARDBP, and OPTN (Brettschneider et al., 2013, 2014; Lorente Pons et al., 2020; Nolan et al., 2020). In the gray matter of the anterior horn, the amount of GCI pathology was reported to correlate with the severity of neuronal TDP43 pathology and neuronal loss (Brettschneider et al., 2014), and in the precentral and middle frontal gyrus, the severity of GCI pathology was reported to correlate with neuronal TDP43 pathology (Lorente Pons et al., 2020). Moreover, GCIs may be more abundant than neuronal pathology and might even precede neuronal TDP-43 pathology, suggesting that particularly gray matter oligodendroglial involvement may be an early event in the disease process (Brettschneider et al., 2014; Nolan et al., 2020). Notably, GCI pathology was mainly observed in oligodendroglia with potential close axonal contacts, whereas satellite cells located around the soma of neurons with TDP-43 pathology remain rather uninvolved (Brettschneider et al., 2013, 2014). In the context of FTLD_TDP, a recent detailed analysis on the subcortical TDP-43 pathology among FTLD-TDP subtypes demonstrated the presence of moderate to frequent GCI pathology as a highly discriminatory feature to separate FTLDTDP type B (including sporadic and genetic cases with C9orf72 


\begin{tabular}{cc} 
A & $\begin{array}{c}\text { Frequency of GCI } \\
\text { pathology }\end{array}$ \\
\hline ALS-TDP & $+/+++$ \\
\hline FTLD-TDP & $-/+$ \\
Type A & $++/++$ \\
Type B & 0 \\
Type C & 0 \\
Type D & +++ \\
Type E & \\
\hline ALS-FUS & $++/+++$ \\
Pattern 1 & $-/+$ \\
Pattern 2 & \\
\hline FTLD-FUS & $+/++$ \\
aFTLD-U & $+/++$ \\
NIFID & $+/++$ \\
BIBD & \\
\hline
\end{tabular}
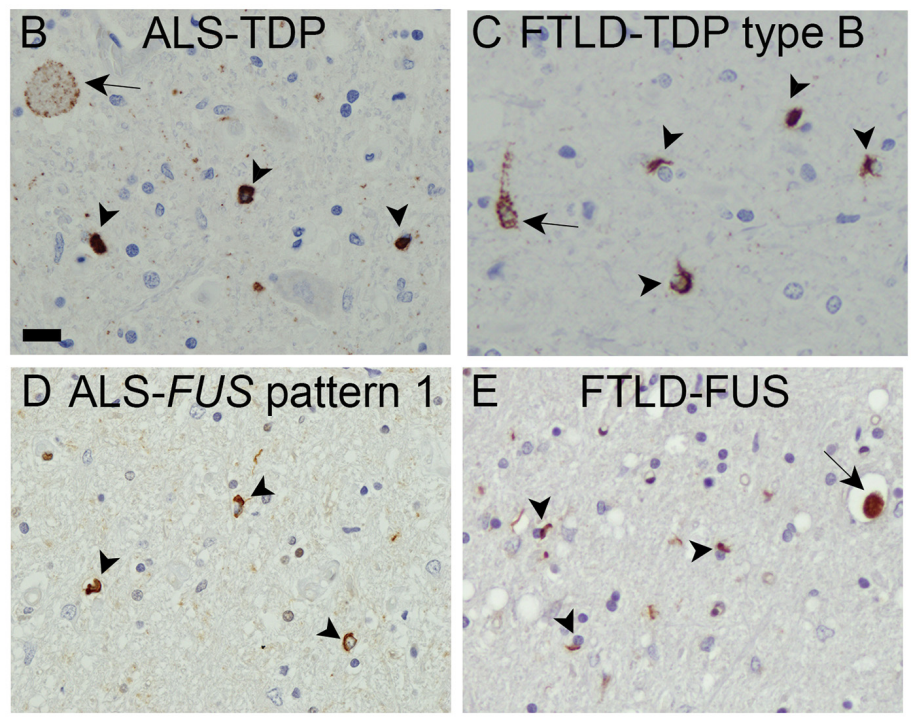

FIGURE 1 | Oligodendroglial pathology in TDP-43 and FUS proteinopathies. (A) Glial cytoplasmic inclusions (GCI) are a characteristic feature with presence in variable amounts in affected brain regions in ALS - TDP, in subtypes of FTLD - TDP (particularly types B,E), subtypes of ALS - FUS (particularly in pattern 1 associated with adult onset and longer disease duration), and all distinct entities of FTLD-FUS, including aFTLD-U, basophilic inclusion body disease, and neuronal intermediate filament inclusion disease. Semiquantitative scores: 0 , absent;+, rare;++, moderate; +++, abundant. (B-E) Immunohistochemistry of human postmortem tissues with pTDP-43 S409/410 antibody (Neumann et al., 2009a) demonstrating numerous GCl (arrowheads) in spinal cord of ALS-TDP case (B) and frontal cortex of FTLD - TDP type B case (C), and FUS (Proteintech) immunohistochemistry demonstrating GCl in spinal cord of ALS-FUS case with pattern 1 pathology (D) and basal ganglia of FTLD-FUS (basophilic inclusion body disease) case (E) in addition to neuronal cytoplasmic inclusions (arrow). Scale bar in panel $\mathbf{B}=20 \mu \mathrm{m}$.

mutations) from other FTLD-TDP types (Mackenzie and Neumann, 2020). Moreover, an association between abundant GCI pathology and shorter disease duration was reported for type E cases (Lee et al., 2017). Thus, oligodendroglial dysfunction might particularly contribute to ALS-TDP and FTLD-TDP type $\mathrm{B}$ and $\mathrm{E}$ pathogenesis. Given the increasing evidence suggesting the existence of distinct TDP-43 strains that might propagate in a prion-like manner as a molecular basis for the distinct clinical and pathological TDP-43 proteinopathies (Kawakami et al., 2019; Neumann et al., 2021), it is tempting to speculate that ALS-TDP and FTLD types B and E might share common TDP-43 strains with a specific cellular tropism for oligodendrocytes.

FUS proteinopathies include ALS cases with FUS mutations and three conditions presenting with FTD and/or a movement disorder, including atypical FTLD-U, neuronal intermediate filament inclusion disease, and basophilic inclusion body disease. GCI pathology is a common feature in all distinct FTLDFUS entities (Mackenzie et al., 2011b). For ALS-FUS, two broad distinct neuropathological patterns based on the frequency, morphology, and distribution pattern of distinct FUS-positive inclusion types can be delineated that correlate with the clinical phenotype and severity of the functional consequence of specific FUS mutations (Mackenzie et al., 2011a). Notably, anatomically widespread moderate/abundant numbers of GCI with a strong correlation between the number of GCI and NCI in the affected neuroanatomical region are a highly characteristic feature for pattern 1, which is associated with longer disease duration and later disease onset (Mackenzie et al., 2011a).

Although the origin of cells with GCI in TDP-43 and FUS proteinopathies as oligodendroglia has been validated in several studies using various markers for immature and mature oligodendroglial lineage cells, including Olig2 (Nolan et al., 2020), TPPP/p25 (Rohan et al., 2014; Fatima et al., 2015), and 2',3'-cyclic-nucleotide $3^{\prime}$-phosphodiesterase (Mackenzie et al., 2011a), it remains to be determined whether specific subtypes of oligodendroglial cells are more prone to develop GCI than others, an issue currently also hampered by the limited specificity/sensitivity of available antibodies in human postmortem tissues.

In addition to neuropathological findings, further evidence for a role of oligodendroglia in ALS/FTD is provided from genetic studies that have identified the oligodendrocyte-specific gene $M O B P$ as a risk factor for ALS (van Rheenen et al., 2016) and a risk variant of $M O B P$ to be associated with more severe white matter degeneration and a clinically more aggressive form of FTD (Irwin et al., 2014). However, this could not be verified in a subanalysis of autopsy-confirmed FTLD-TDP cases in this cohort, although the total numbers of cases might have been too small (Irwin et al., 2014). More recently, applying a polygenic risk score approach with combined analysis of genomewide association study and single-nucleus RNA sequencing datasets uncovered the association of a distinct subpopulation of oligodendrocytes to ALS risks (Saez-Atienzar et al., 2021). 


\section{POTENTIAL MECHANISMS OF OLIGODENDROGLIAL DYSFUNCTION IN THE ALS/FTLD SPECTRUM}

Although oligodendrocyte biology has been extensively reviewed elsewhere (Butt et al., 2019), some key aspects relevant in the context of ALS/FTLD pathogenesis are worth mentioning here. Briefly, oligodendrocytes differentiate from oligodendrocytes precursor cells (OPCs) during embryonic and postnatal development in rodents (Takebayashi and Ikenaka, 2015) and humans (Czepiel et al., 2015). However, the central nervous system hosts an OPC pool, which constitutes 5-10\% of glial cells and which retains the potential to generate new mature oligodendrocytes as well as astrocytes throughout the entire life (Fletcher et al., 2021). Recently, single-cell RNA sequencing analysis revealed that differentiation is a graded process leading to the generation of distinct subtypes of mature oligodendrocytes (Marques et al., 2016), whose most thoroughly characterized functional task is the formation and the maintenance of myelin wraps around the axons to ensure saltatory conduction. Furthermore, oligodendrocytes support the axons by providing trophic and metabolic support. Specifically, different groups identified lactate as an energy source that is transferred from oligodendrocytes to axons (Funfschilling et al., 2012; Lee et al., 2012). Finally, oligodendrocytes engage in bidirectional communications with neurons by responding to glutamatergic signaling with the neuroprotective release of extracellular vesicles such as exosomes (Fruhbeis et al., 2013, 2020; Mukherjee et al., 2020).

Because cells with TDP-43 or FUS-positive GCI displays a noticeable depletion of the physiological nuclear immunoreactivity, one can speculate that affected oligodendrocytes develop a pathological phenotype resulting from the combined action of the loss of nuclear/cytoplasmic functions and the gain of toxicity induced by the abnormal cytoplasmic expression. However, crucial questions to be resolved are as follows: What are the functional consequences, and how might oligodendroglial dysfunction contribute to degeneration? What causes/triggers TDP-43/FUS aggregation in oligodendrocytes?

Different aspects are discussed in the following and illustrated in Figure 2.

\section{Oligodendrocytes Precursor Cells Differentiation}

In mice, both Tardbp and Fus are highly expressed in OPCs, and their expression decreases with oligodendrocyte maturation (Zhang et al., 2014) ${ }^{1}$, prompting the speculation that TDP43/FUS might play important roles in those cells. Neural progenitor cells derived from an ALS patient with a TARDBP mutation differentiate in vitro to mature oligodendrocyte as efficiently as cells derived from non-ALS cases or other sALS and fALS patients, including those with C9orf72 mutation, whereas

${ }^{1}$ https://www.brainrnaseq.org/ mutant FUS carriers were not investigated (Ferraiuolo et al., 2016; Livesey et al., 2016). However, it has to be noted that these models do not recapitulate inclusion body formation with nuclear depletion of TDP-43. Unfortunately, no OPC-specific knockout models nor transgenic mice selectively expressing mutant versions of either protein have yet been generated, and therefore, we currently lack in vivo information as to how the loss of TDP-43/FUS might regulate OPC functions and their ability to differentiate into mature oligodendrocytes. This is of outstanding importance because the generation of new oligodendrocytes not only occurs physiologically in response to neuronal activity and it is important for learning and memory (Fletcher et al., 2021), but it is also key to restore myelin upon injury (Biname et al., 2021).

\section{Mature Oligodendrocytes Survival}

To investigate the role of TDP-43 and FUS in mature oligodendrocytes in vivo, Tardbp (Wang et al., 2018) or Fus (Guzman et al., 2020) was depleted from cells expressing $2^{\prime}, 3^{\prime}$-cyclic-nucleotide $3^{\prime}$-phosphodiesterase. Interestingly, TDP43 depletion leads to their demise and a compensatory hyperproliferation and differentiation of OPCs in the white matter in transgenic mice (Wang et al., 2018). In contrast, Fus ablation does not lead to oligodendrocyte death (Guzman et al., 2020), thus suggesting that FUS functions could be compensated by other RNA-binding proteins, whereas TDP-43 is nondispensable in mice. These findings should be complemented by generating transgenic mice selectively expressing mutant TDP-43 or mutant FUS in mature oligodendrocytes to investigate whether mutations recapitulate the knockout phenotype or whether they induce novel toxic functions.

\section{Myelination}

TDP-43 and FUS are likely implicated in several aspects of myelination. Firstly, cross-linking immunoprecipitation sequencing experiments discovered that TDP- 43 binds to the mRNAs from key genes for myelination such as Plp1, Mbp, Mog, and Mag (Wang et al., 2018), whereas FUS binds to MBP (Hoell et al., 2011) and Mal (Lagier-Tourenne et al., 2012). Secondly, FUS depletion leads to Mobp alternative splicing (Lagier-Tourenne et al., 2012), whereas either TDP-43 or FUS depletion leads to alternative splicing of Mag exon 8 (LagierTourenne et al., 2012; Kapeli et al., 2016; Figure 2), although with opposite effects, thus corroborating the hypothesis that these proteins affect oligodendrocytes with distinct modalities. Finally, although most myelin-associated transcripts are translated in the perinuclear region and then transported as mature proteins, MBP protein synthesis occurs distally, at the sites of active myelination, thus requiring the transport of $M b p$ mRNA in translationally silent RNA granules (Muller et al., 2013), membrane-less organelles arising from the liquid-liquid phase separation of intrinsically disordered RNA/DNA binding proteins such as FUS and TDP-43 (Portz et al., 2021; Figure 2). As several lines of evidence indicate that both TDP-43 and FUS are implicated in mRNA particle formation and mRNA trafficking in neurons (Thelen and Kye, 2019), one could 


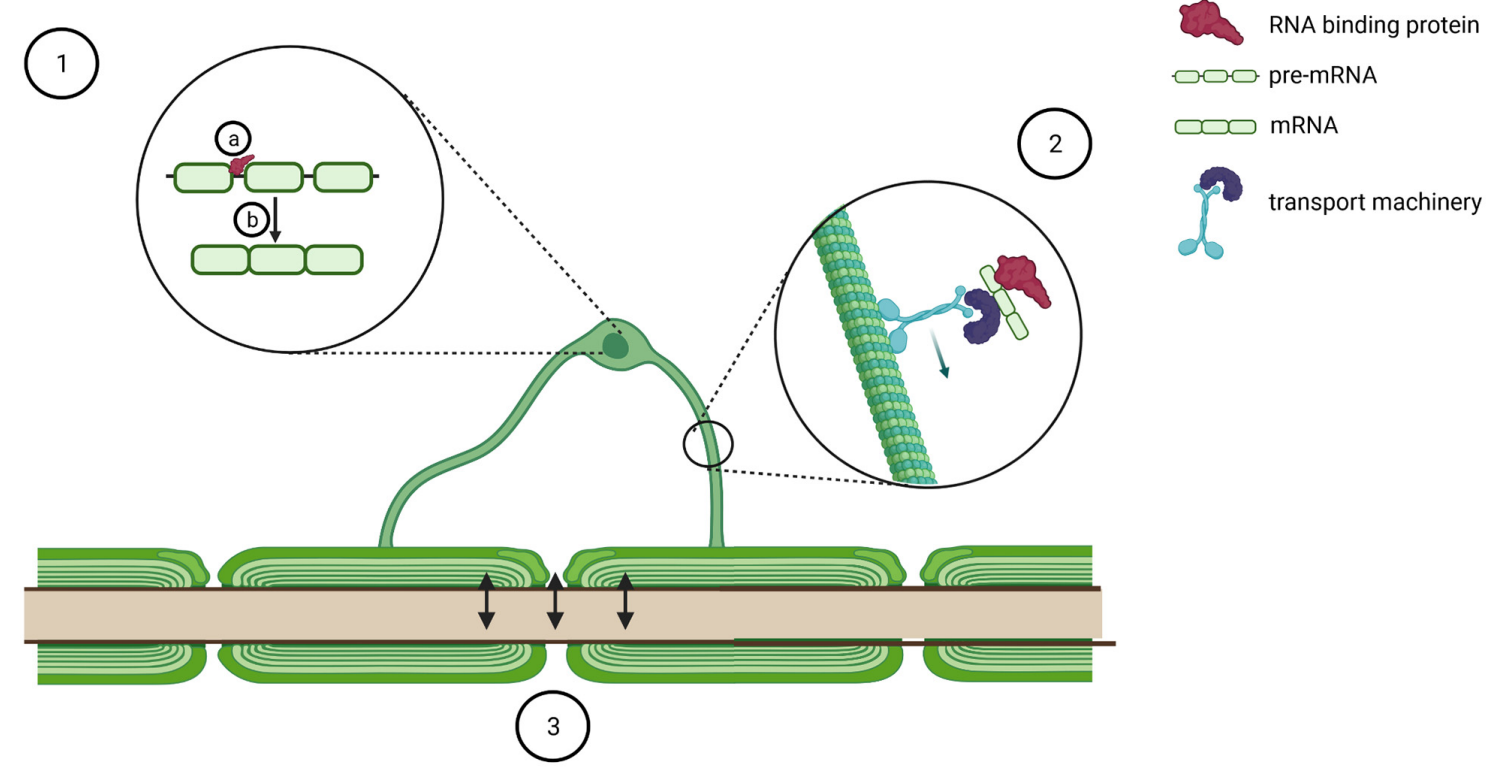

FIGURE 2 | Schematic of potential mechanisms of oligodendrocyte dysfunction in pathogenesis of TDP-43/FUS proteinopathies. TDP-43 and FUS pathology in oligodendrocytes might modulate oligodendrocyte biology by binding to several transcripts (1a) and regulating pre-mRNA splicing (1b). Furthermore, it might affect transport of key RNA transcripts such as Mbp (2) and perturb bidirectional communication with axons (3). Created with BioRender.com.

speculate that they might contribute to the same process in oligodendrocytes. In particular, $M b p$ transport is a tightly regulated process that requires the distinct action of several RNA binding proteins, including hnRNPA2B1 (Raju et al., 2008), hnRNP K (Torvund-Jensen et al., 2014), and fragile $\mathrm{X}$ mental retardation protein (Doll et al., 2020). Intriguingly, TDP-43 is a well-characterized interactor of hnRNPA2 (Buratti et al., 2005; He et al., 2014; Romano et al., 2014; Chiang et al., 2020), hnRNP K (Moujalled et al., 2015, 2017), and fragile X mental retardation protein (Blokhuis et al., 2016; Majumder et al., 2016; Chu et al., 2019), which is also a FUS interacting partner (Blokhuis et al., 2016; He and Ge, 2017). In addition, indirect mechanisms could also tune $M b p$ transport. To this end, it is worth mentioning that FUS and TDP-43 regulate the alternative splicing and the stability of Mapt (Orozco et al., 2012; Gu et al., 2017a,b; Ishigaki et al., 2017; Wu et al., 2021), the gene coding for tau, a protein involved in the regulation of the microtubular network and necessary for $M b p$ mRNA transport as well as for the correct maturation and myelination process of oligodendrocytes (Seiberlich et al., 2015). Intriguingly, ubiquitous $\mathrm{TDP}-43^{\mathrm{Q} 331 \mathrm{~K}}$ knock-in mice can be stratified into two populations on the basis of their behavioral impairment and affected mice display altered Mapt splicing pattern, whereas unaffected animals upregulate genes regulating myelination (White et al., 2018). Taken together, these data indicate oligodendrocytes as key disease modifiers and suggest that impaired focal delivery of $M b p$ could be a contributor to TDP-43 and FUS proteinopathies. In line with this, a recent paper reports that the relative protein expression of MBP versus PLP1 is reduced in spinal cord corticospinal tract white matter of ALS patients (Lorente Pons et al., 2020).

\section{Oligodendrocyte-Neuron and Oligodendrocyte-Astrocyte Interplay}

In addition to myelination, oligodendrocytes provide trophic support to neurons (Duncan et al., 2021), a task that becomes defective in patient-derived oligodendrocytes. Indeed, neurons cocultured with ALS oligodendrocytes display reduced survival, even in the absence of obvious differentiation impairment (Ferraiuolo et al., 2016). Conversely, no evidence of neurotoxicity has yet been presented in mouse models where TDP-43 (Wang et al., 2018) or FUS (Guzman et al., 2020) has been depleted from oligodendrocytes. There are different possibilities to explain such discrepancy. Firstly, mutant TDP-43 might trigger oligodendrocytes dysfunction through gain of toxicity, and to date, selective expression of mutant TDP-43 or FUS in oligodendrocytes has not been investigated in transgenic mice. Alternatively, oligodendrocyte biology in animal models might not adequately recapitulate their functions in humans, an interpretation supported by single-nucleus RNA sequencing studies that revealed that only some human oligodendrocyte subpopulations have a counterpart in the rodent brain (Hodge et al., 2019). Moreover, a study comparing the transcriptome of neurons and oligodendrocytes from human and non-human primates unveiled that oligodendrocytes display a higher degree of evolution than neurons (Berto et al., 2019). Taken together, these findings urge us to interpret data gathered from mouse models with caution and underline the need for the development of human-derived models, such as brain organoids (Shaker et al., 2021), to explore both cell-autonomous and non-cellautonomous aspects of oligodendrocyte biology.

Mechanistically, oligodendrocytes support neurons by releasing lactate (Funfschilling et al., 2012; Lee et al., 2012; 
Figure 2), and reduction of both oligodendrocyte and neuronal lactate transporters has been reported in the motor cortex of ALS patients (Lee et al., 2012). However, in coculture experiments, reduced lactate release from ALS/FTD oligodendrocytes only partially explains their impaired ability to support neuronal viability (Ferraiuolo et al., 2016), arguing that other mechanisms are likely contributing to the pathogenic mechanism. To this end, an interesting hypothesis arises from the trophic effect that glutamate-dependent oligodendrocyte-derived exosomes play on axons (Fruhbeis et al., 2013, 2020; Mukherjee et al., 2020) as both TDP-43 (Iguchi et al., 2016) and FUS (Kamelgarn et al., 2016) have been identified as components of exosomes purified from different cell culture supernatants. Moreover, in the plasma of sALS patients, both TDP-43 and FUS can be detected in microvesicles (Sproviero et al., 2018), and the accumulation of TDP-43 in plasma exosomes is currently under investigation as a potential progression biomarker in ALS (Chen et al., 2020). Taken together, these findings prompt several questions, which would deserve ad hoc investigations: which is the cellular source of TDP-43 and FUS containing exosomes? Are exosome biogenesis and release impaired? Is the composition of exosome payload perturbed in oligodendrocytes derived from ALS and FTLD patients? Finally, oligodendrocytes protect axons from the depolarizing effect of extracellular potassium accumulation by expressing the potassium channels Kir4.1 (Schirmer et al., 2018). Intriguingly, the same channels are also expressed on astrocytes, where they modulate the morphology and the electrophysiological properties of fast $\alpha$-motor neurons (Kelley et al., 2018). Because ALS patient-derived astrocytes display reduced expression of Kir4.1 (Kelley et al., 2018), it would be of outstanding interest to learn whether oligodendrocytes might show the same defect.

Complementary, oligodendrocyte dysfunction in ALS/FTLD might not be exclusively cell-autonomous, but it could arise from aberrant signaling coming from other glial cell populations. In particular, the genetic ablation of Tardbp in astrocytes switches their transcriptome and that of the surrounding microglia toward an inflammatory status in mice (Peng et al., 2020). Furthermore, the spinal cord of those animals displays a reduced number of mature oligodendrocytes in the gray matter (Peng et al., 2020).

\section{MECHANISMS OF SELECTIVE VULNERABILITY OF OLIGODENDROCYTES IN AMYOTROPHIC LATERAL SCLEROSIS/FRONTOTEMPORAL LOBAR DEGENERATION SPECTRUM}

The neuropathological analysis showing that TDP-43 or FUS aggregates are restricted to subsets of neurons and oligodendrocytes prompts the question as to why these cell types are exquisitely vulnerable to the formation of inclusions. Although this topic is still under-investigated, one can speculate that oligodendrocyte susceptibility might arise from their high energy demand required for myelination (Tepavcevic, 2021) and active mRNA transport over long distances, exposing them to mitochondrial dysfunction and oxidative stress. In cultured oligodendrocytes, oxidative insult induces stress granules, membrane-less organelles that transiently store translationally silenced mRNA, including Mbp (Wang et al., 2010), and are thought to act as nucleation events for inclusion body formation in TDP-43/FUS-proteinopathies upon aberrant dynamics of assembly and disassembly (Baradaran-Heravi et al., 2020). Intriguingly, edaravone, a recently approved drug for the treatment of ALS, has been shown to protect oligodendrocytes from oxidative insults (Ueno et al., 2009; Miyamoto et al., 2013).

A complementary hypothesis might be that oligodendrocytes have a reduced proteostatic capacity because they display increased vulnerability to proteasome impairment when compared with other glial cells (Goldbaum et al., 2006). Moreover, the inactivation of the unfolded protein response leads to subsequent autophagy impairment and oligodendrocyte toxicity (Stone et al., 2020), and in the motor cortex of ALS cases, the expression of unfolded protein response target genes was found to be associated with oligodendrocytes (Montibeller et al., 2020). More specifically, TDP-43 binds to the von Hippel Lindau protein (VHL), an adapter that targets its interaction partners to the ubiquitination machinery for subsequent degradation (Uchida et al., 2016). TDP-43 depletion leads to VHL overexpression that has the paradoxical effect of promoting TDP-43 inclusion formation (Uchida et al., 2016). One could therefore speculate that an initial insult leads to stress granule formation, which in turn causes an early depletion of TDP43 from the nucleus and upregulation of VHL. This event would hyper-stabilize TDP-43 granules converting them into pathological aggregates, which would induce further cellular stress and therefore closing a harmful vicious cycle.

\section{CONCLUSION}

In summary, investigations so far have provided substantial evidence for a potential role of oligodendroglial dysfunction in ALS/FTLD. However, more detailed studies on the physiological roles of TDP-43 and FUS in the complex functions of oligodendroglial lineage cells and the determination of molecular changes during the disease process are required to further dissect the significance of specific alterations in disease pathogenesis. This might pave the way for new therapeutic approaches in ALS/FTLD by, e.g., boosting oligodendrocyte metabolism and functions.

\section{AUTHOR CONTRIBUTIONS}

$\mathrm{CV}$ and $\mathrm{MN}$ researched literature, conceived the presented concepts, and wrote the manuscript. Both authors contributed to the article and approved the submitted version.

\section{FUNDING}

This work was supported by grants from the German Research Foundation (NE 850/4-1), the NOMIS Foundation, and by a generous donor who wishes to remain anonymous. 


\section{REFERENCES}

Arai, T., Hasegawa, M., Akiyama, H., Ikeda, K., Nonaka, T., Mori, H., et al. (2006). TDP-43 is a component of ubiquitin-positive tau-negative inclusions in frontotemporal lobar degeneration and amyotrophic lateral sclerosis. Biochem. Biophys. Res. Commun. 351, 602-611. doi: 10.1016/j.bbrc.2006.10.093

Baradaran-Heravi, Y., Van Broeckhoven, C., and van der Zee, J. (2020). Stress granule mediated protein aggregation and underlying gene defects in the FTDALS spectrum. Neurobiol. Dis. 134, 104639. doi: 10.1016/j.nbd.2019.104639

Berto, S., Mendizabal, I., Usui, N., Toriumi, K., Chatterjee, P., Douglas, C., et al. (2019). Accelerated evolution of oligodendrocytes in the human brain. Proc. Natl. Acad. Sci. U.S.A. 116, 24334-24342. doi: 10.1073/pnas.1907982116

Biname, F., Pham-Van, L. D., and Bagnard, D. (2021). Manipulating oligodendrocyte intrinsic regeneration mechanism to promote remyelination. Cell. Mol. Life Sci. 78, 5257-5273. doi: 10.1007/s00018-021-03852-4

Blokhuis, A. M., Koppers, M., Groen, E. J. N., van den Heuvel, D. M. A., Dini Modigliani, S., Anink, J. J., et al. (2016). Comparative interactomics analysis of different ALS-associated proteins identifies converging molecular pathways. Acta Neuropathol. 132, 175-196. doi: 10.1007/s00401-016-1575-8

Bowden, H. A., and Dormann, D. (2016). Altered mRNP granule dynamics in FTLD pathogenesis. J Neurochem 138(Suppl. 1), 112-133. doi: 10.1111/jnc. 13601

Brettschneider, J., Arai, K., Del Tredici, K., Toledo, J. B., Robinson, J. L., Lee, E. B., et al. (2014). TDP-43 pathology and neuronal loss in amyotrophic lateral sclerosis spinal cord. Acta Neuropathol. 128, 423-437. doi: 10.1007/s00401-0141299-6

Brettschneider, J., Del Tredici, K., Toledo, J. B., Robinson, J. L., Irwin, D. J., Grossman, M., et al. (2013). Stages of pTDP-43 pathology in amyotrophic lateral sclerosis. Ann. Neurol. 74, 20-38. doi: 10.1002/ana.23937

Buratti, E. (2021). Trends in understanding the pathological roles of TDP-43 and FUS proteins. Adv. Exp. Med. Biol. 1281, 243-267. doi: 10.1007/978-3-03051140-1_15

Buratti, E., Brindisi, A., Giombi, M., Tisminetzky, S., Ayala, Y. M., and Baralle, F. E. (2005). TDP-43 binds heterogeneous nuclear ribonucleoprotein A/B through its C-terminal tail: an important region for the inhibition of cystic fibrosis transmembrane conductance regulator exon 9 splicing. J. Biol. Chem. 280, 37572-37584. doi: 10.1074/jbc.M505557200

Burrell, J. R., Halliday, G. M., Kril, J. J., Ittner, L. M., Gotz, J., Kiernan, M. C., et al. (2016). The frontotemporal dementia-motor neuron disease continuum. Lancet 388, 919-931. doi: 10.1016/S0140-6736(16)00737-6

Butt, A. M., Papanikolaou, M., and Rivera, A. (2019). Physiology of Oligodendroglia. Adv. Exp. Med. Biol. 1175, 117-128. doi: 10.1007/978981-13-9913-8_5

Chen, P. C., Wu, D., Hu, C. J., Chen, H. Y., Hsieh, Y. C., and Huang, C. C. (2020) Exosomal TAR DNA-binding protein-43 and neurofilaments in plasma of amyotrophic lateral sclerosis patients: a longitudinal follow-up study. J. Neurol. Sci. 418:117070. doi: 10.1016/j.jns.2020.117070

Chiang, W. C., Lee, M. H., Chen, T. C., and Huang, J. R. (2020). Interactions between the intrinsically disordered regions of hnRNP-A2 and TDP-43 accelerate TDP-43's conformational transition. Int. J. Mol. Sci. 21:5930. doi: $10.3390 /$ ijms 21165930

Chu, J. F., Majumder, P., Chatterjee, B., Huang, S. L., and Shen, C. J. (2019). TDP-43 regulates coupled dendritic mRNA transport-translation processes in co-operation with FMRP and Staufen1. Cell Rep. 29, 3118-3133.e3116. doi: 10.1016/j.celrep.2019.10.061

Czepiel, M., Boddeke, E., and Copray, S. (2015). Human oligodendrocytes in remyelination research. Glia 63, 513-530. doi: 10.1002/glia.22769

Doll, C. A., Yergert, K. M., and Appel, B. H. (2020). The RNA binding protein fragile X mental retardation protein promotes myelin sheath growth. Glia 68 495-508. doi: 10.1002/glia.23731

Duncan, G. J., Simkins, T. J., and Emery, B. (2021). Neuron-Oligodendrocyte interactions in the structure and integrity of axons. Front. Cell Dev. Biol. 9:653101. doi: 10.3389/fcell.2021.653101

Ederle, H., and Dormann, D. (2017). TDP-43 and FUS en route from the nucleus to the cytoplasm. FEBS Lett. 591, 1489-1507. doi: 10.1002/1873-3468.12646

Ettle, B., Schlachetzki, J. C. M., and Winkler, J. (2016). Oligodendroglia and myelin in neurodegenerative diseases: more than just bystanders? Mol. Neurobiol. 53, 3046-3062. doi: 10.1007/s12035-015-9205-3
Fatima, M., Tan, R., Halliday, G. M., and Kril, J. J. (2015). Spread of pathology in amyotrophic lateral sclerosis: assessment of phosphorylated TDP-43 along axonal pathways. Acta Neuropathol. Commun. 3:47. doi: 10.1186/s40478-0150226-y

Ferraiuolo, L., Meyer, K., Sherwood, T. W., Vick, J., Likhite, S., Frakes, A., et al. (2016). Oligodendrocytes contribute to motor neuron death in ALS via SOD1dependent mechanism. Proc. Natl. Acad. Sci. U.S.A. 113, E6496-E6505. doi: $10.1073 /$ pnas. 1607496113

Fletcher, J. L., Makowiecki, K., Cullen, C. L., and Young, K. M. (2021). Oligodendrogenesis and myelination regulate cortical development, plasticity and circuit function. Semin. Cell Dev. Biol. S1084-9521(21)00064-1. doi: 10. 1016/j.semcdb.2021.03.017

Fruhbeis, C., Frohlich, D., Kuo, W. P., Amphornrat, J., Thilemann, S., Saab, A. S., et al. (2013). Neurotransmitter-triggered transfer of exosomes mediates oligodendrocyte-neuron communication. PLoS Biol. 11:e1001604. doi: 10.1371/ journal.pbio. 1001604

Fruhbeis, C., Kuo-Elsner, W. P., Muller, C., Barth, K., Peris, L., Tenzer, S., et al. (2020). Oligodendrocytes support axonal transport and maintenance via exosome secretion. PLoS Biol. 18:e3000621. doi: 10.1371/journal.pbio.3000621

Funfschilling, U., Supplie, L. M., Mahad, D., Boretius, S., Saab, A. S., Edgar, J., et al. (2012). Glycolytic oligodendrocytes maintain myelin and long-term axonal integrity. Nature 485, 517-521. doi: 10.1038/nature11007

Geser, F., Brandmeir, N. J., Kwong, L. K., Martinez-Lage, M., Elman, L., McCluskey, L., et al. (2008). Evidence of multisystem disorder in whole-brain map of pathological TDP-43 in amyotrophic lateral sclerosis. Arch. Neurol. 65, 636641. doi: 10.1001/archneur.65.5.636

Goldbaum, O., Vollmer, G., and Richter-Landsberg, C. (2006). Proteasome inhibition by MG-132 induces apoptotic cell death and mitochondrial dysfunction in cultured rat brain oligodendrocytes but not in astrocytes. Glia 53, 891-901. doi: 10.1002/glia.20348

Gu, J., Chen, F., Iqbal, K., Gong, C. X., Wang, X., and Liu, F. (2017a). Transactive response DNA-binding protein 43 (TDP-43) regulates alternative splicing of tau exon 10: implications for the pathogenesis of tauopathies. J. Biol. Chem. 292, 10600-10612. doi: 10.1074/jbc.M117.783498

Gu, J., Wu, F., Xu, W., Shi, J., Hu, W., Jin, N., et al. (2017b). TDP-43 suppresses tau expression via promoting its mRNA instability. Nucleic Acids Res. 45, 6177-6193. doi: 10.1093/nar/gkx175

Guzman, K. M., Brink, L. E., Rodriguez-Bey, G., Bodnar, R. J., Kuang, L., Xing, B., et al. (2020). Conditional depletion of Fus in oligodendrocytes leads to motor hyperactivity and increased myelin deposition associated with Akt and cholesterol activation. Glia 68, 2040-2056. doi: 10.1002/glia. 23825

Hardiman, O., Al-Chalabi, A., Chio, A., Corr, E. M., Logroscino, G., Robberecht, W., et al. (2017). Amyotrophic lateral sclerosis. Nat. Rev. Dis. Primers 3:17071. doi: 10.1038/nrdp.2017.71

He, F., Krans, A., Freibaum, B. D., Taylor, J. P., and Todd, P. K. (2014). TDP43 suppresses CGG repeat-induced neurotoxicity through interactions with HnRNP A2/B1. Hum. Mol. Genet. 23, 5036-5051. doi: 10.1093/hmg/ddu216

He, Q., and Ge, W. (2017). The tandem Agenet domain of fragile X mental retardation protein interacts with FUS. Sci. Rep. 7:962. doi: 10.1038/s41598017-01175-8

Hodge, R. D., Bakken, T. E., Miller, J. A., Smith, K. A., Barkan, E. R., Graybuck, L. T., et al. (2019). Conserved cell types with divergent features in human versus mouse cortex. Nature 573, 61-68. doi: 10.1038/s41586-019-1506-7

Hoell, J. I., Larsson, E., Runge, S., Nusbaum, J. D., Duggimpudi, S., Farazi, T. A., et al. (2011). RNA targets of wild-type and mutant FET family proteins. Nat. Struct. Mol. Biol. 18, 1428-1431. doi: 10.1038/nsmb.2163

Iguchi, Y., Eid, L., Parent, M., Soucy, G., Bareil, C., Riku, Y., et al. (2016). Exosome secretion is a key pathway for clearance of pathological TDP-43. Brain 139(Pt 12), 3187-3201. doi: 10.1093/brain/aww237

Irwin, D. J., McMillan, C. T., Suh, E., Powers, J., Rascovsky, K., Wood, E. M., et al. (2014). Myelin oligodendrocyte basic protein and prognosis in behavioralvariant frontotemporal dementia. Neurology 83, 502-509. doi: 10.1212/WNL. 0000000000000668

Ishigaki, S., Fujioka, Y., Okada, Y., Riku, Y., Udagawa, T., Honda, D., et al. (2017). Altered tau isoform ratio caused by loss of FUS and SFPQ function leads to FTLD-like phenotypes. Cell Rep. 18, 1118-1131. doi: 10.1016/j.celrep.2017.01. 013 
Kamelgarn, M., Chen, J., Kuang, L., Arenas, A., Zhai, J., Zhu, H., et al. (2016). Proteomic analysis of FUS interacting proteins provides insights into FUS function and its role in ALS. Biochim. Biophys. Acta 1862, 2004-2014. doi: 10.1016/j.bbadis.2016.07.015

Kapeli, K., Pratt, G. A., Vu, A. Q., Hutt, K. R., Martinez, F. J., Sundararaman, B., et al. (2016). Distinct and shared functions of ALS-associated proteins TDP43, FUS and TAF15 revealed by multisystem analyses. Nat. Commun. 7:12143. doi: $10.1038 /$ ncomms 12143

Kawakami, I., Arai, T., and Hasegawa, M. (2019). The basis of clinicopathological heterogeneity in TDP-43 proteinopathy. Acta Neuropathol. 138, 751-770. doi: 10.1007/s00401-019-02077-x

Kelley, K. W., Ben Haim, L., Schirmer, L., Tyzack, G. E., Tolman, M., Miller, J. G., et al. (2018). Kir4.1-dependent astrocyte-fast motor neuron interactions are required for peak strength. Neuron 98, 306-319.e307. doi: 10.1016/j.neuron. 2018.03.010

Kwiatkowski, T. J. Jr., Bosco, D. A., Leclerc, A. L., Tamrazian, E., Vanderburg, C. R., Russ, C., et al. (2009). Mutations in the FUS/TLS gene on chromosome 16 cause familial amyotrophic lateral sclerosis. Science 323, 1205-1208. doi: 10.1126/science. 1166066

Lagier-Tourenne, C., Polymenidou, M., Hutt, K. R., Vu, A. Q., Baughn, M., Huelga, S. C., et al. (2012). Divergent roles of ALS-linked proteins FUS/TLS and TDP43 intersect in processing long pre-mRNAs. Nat. Neurosci. 15, 1488-1497. doi: $10.1038 / \mathrm{nn} .3230$

Lee, E. B., Porta, S., Michael Baer, G., Xu, Y., Suh, E., Kwong, L. K., et al. (2017). Expansion of the classification of FTLD-TDP: distinct pathology associated with rapidly progressive frontotemporal degeneration. Acta Neuropathol. 134, 65-78. doi: 10.1007/s00401-017-1679-9

Lee, Y., Morrison, B. M., Li, Y., Lengacher, S., Farah, M. H., Hoffman, P. N., et al. (2012). Oligodendroglia metabolically support axons and contribute to neurodegeneration. Nature 487, 443-448. doi: 10.1038/nature11314

Livesey, M. R., Magnani, D., Cleary, E. M., Vasistha, N. A., James, O. T., Selvaraj, B. T., et al. (2016). Maturation and electrophysiological properties of human pluripotent stem cell-derived oligodendrocytes. Stem Cells 34, 1040-1053. doi: $10.1002 /$ stem.2273

Lorente Pons, A., Higginbottom, A., Cooper-Knock, J., Alrafiah, A., Alofi, E., Kirby, J., et al. (2020). Oligodendrocyte pathology exceeds axonal pathology in white matter in human amyotrophic lateral sclerosis. J. Pathol. 251, 262-271. doi: 10.1002/path.5455

Lu, Q. R., Sun, T., Zhu, Z., Ma, N., Garcia, M., Stiles, C. D., et al. (2002). Common developmental requirement for Olig function indicates a motor neuron/oligodendrocyte connection. Cell 109, 75-86. doi: 10.1016/s00928674(02)00678-5

Mackenzie, I. R. A., Ansorge, O., Strong, M., Bilbao, J., Zinman, L., Ang, L. C., et al. (2011a). Pathological heterogeneity in amyotrophic lateral sclerosis with FUS mutations: two distinct patterns correlating with disease severity and mutation. Acta Neuropathol. 122, 87-98. doi: 10.1007/s00401-011-0838-7

Mackenzie, I. R. A., Munoz, D. G., Kusaka, H., Yokota, O., Ishihara, K., Roeber, S., et al. (2011b). Distinct pathological subtypes of FTLD-FUS. Acta Neuropathol. 121, 207-218. doi: 10.1007/s00401-010-0764-0

Mackenzie, I. R., and Neumann, M. (2017). Reappraisal of TDP-43 pathology in FTLD-U subtypes. Acta Neuropathol. 134, 79-96. doi: 10.1007/s00401-0171716-8

Mackenzie, I. R., and Neumann, M. (2020). Subcortical TDP-43 pathology patterns validate cortical FTLD-TDP subtypes and demonstrate unique aspects of C9orf72 mutation cases. Acta Neuropathol. 139, 83-98. doi: 10.1007/s00401019-02070-4

Mackenzie, I. R., Bigio, E. H., Ince, P. G., Geser, F., Neumann, M., Cairns, N. J., et al. (2007). Pathological TDP-43 distinguishes sporadic amyotrophic lateral sclerosis from amyotrophic lateral sclerosis with SOD1 mutations. Ann Neurol. 61, 427-434. doi: 10.1002/ana.21147

Majumder, P., Chu, J. F., Chatterjee, B., Swamy, K. B., and Shen, C. J. (2016). Coregulation of mRNA translation by TDP-43 and Fragile X Syndrome protein FMRP. Acta Neuropathol. 132, 721-738. doi: 10.1007/s00401-016-1603-8

Marques, S., Zeisel, A., Codeluppi, S., van Bruggen, D., Mendanha Falcao, A., Xiao, L., et al. (2016). Oligodendrocyte heterogeneity in the mouse juvenile and adult central nervous system. Science 352, 1326-1329. doi: 10.1126/science.aaf 6463
Miyamoto, N., Maki, T., Pham, L. D., Hayakawa, K., Seo, J. H., Mandeville, E. T., et al. (2013). Oxidative stress interferes with white matter renewal after prolonged cerebral hypoperfusion in mice. Stroke 44, 3516-3521. doi: 10.1161/ STROKEAHA.113.002813

Montibeller, L., Tan, L. Y., Kim, J. K., Paul, P., and de Belleroche, J. (2020). Tissueselective regulation of protein homeostasis and unfolded protein response signalling in sporadic ALS. J. Cell. Mol. Med. 24, 6055-6069. doi: 10.1111/jcmm. 15170

Mot, A. I., Depp, C., and Nave, K. A. (2018). An emerging role of dysfunctional axon-oligodendrocyte coupling in neurodegenerative diseases. Dialogues Clin. Neurosci. 20, 283-292.

Moujalled, D., Grubman, A., Acevedo, K., Yang, S., Ke, Y. D., Moujalled, D. M., et al. (2017). TDP-43 mutations causing amyotrophic lateral sclerosis are associated with altered expression of RNA-binding protein hnRNP K and affect the Nrf2 antioxidant pathway. Hum. Mol. Genet. 26, 1732-1746. doi: 10.1093/ $\mathrm{hmg} / \mathrm{ddx} 093$

Moujalled, D., James, J. L., Yang, S., Zhang, K., Duncan, C., Moujalled, D. M., et al. (2015). Phosphorylation of hnRNP K by cyclin-dependent kinase 2 controls cytosolic accumulation of TDP-43. Hum. Mol. Genet. 24, 1655-1669. doi: 10. 1093/hmg/ddu578

Mukherjee, C., Kling, T., Russo, B., Miebach, K., Kess, E., Schifferer, M., et al. (2020). Oligodendrocytes provide antioxidant defense function for neurons by secreting ferritin heavy chain. Cell Metab. 32, 259-272.e210.

Muller, C., Bauer, N. M., Schafer, I., and White, R. (2013). Making myelin basic protein -from mRNA transport to localized translation. Front. Cell. Neurosci. 7:169. doi: $10.3389 /$ fncel.2013.00169

Munoz, D. G., Neumann, M., Kusaka, H., Yokota, O., Ishihara, K., Terada, S., et al. (2009). FUS pathology in basophilic inclusion body disease. Acta Neuropathol. 118, 617-627. doi: 10.1007/s00401-009-0598-9

Neumann, M., Kwong, L. K., Lee, E. B., Kremmer, E., Flatley, A., Xu, Y., et al. (2009a). Phosphorylation of S409/410 of TDP-43 is a consistent feature in all sporadic and familial forms of TDP-43 proteinopathies. Acta Neuropathol. 117, 137-149. doi: 10.1007/s00401-008-0477-9

Neumann, M., Kwong, L. K., Truax, A. C., Vanmassenhove, B., Kretzschmar, H. A., Van Deerlin, V. M., et al. (2007). TDP-43-positive white matter pathology in frontotemporal lobar degeneration with ubiquitin-positive inclusions. J. Neuropathol. Exp. Neurol. 66, 177-183. doi: 10.1097/01.jnen.0000248554. 45456.58

Neumann, M., Lee, E. B., and Mackenzie, I. R. (2021). Frontotemporal lobar degeneration TDP-43-immunoreactive pathological subtypes: clinical and mechanistic significance. Adv. Exp. Med. Biol. 1281, 201-217. doi: 10.1007/9783-030-51140-1_13

Neumann, M., Rademakers, R., Roeber, S., Baker, M., Kretzschmar, H. A., and Mackenzie, I. R. (2009b). A new subtype of frontotemporal lobar degeneration with FUS pathology. Brain 132(Pt 11), 2922-2931. doi: 10.1093/brain/aw p214

Neumann, M., Roeber, S., Kretzschmar, H. A., Rademakers, R., Baker, M., and Mackenzie, I. R. (2009c). Abundant FUS-immunoreactive pathology in neuronal intermediate filament inclusion disease. Acta Neuropathol. 118, 605 616. doi: 10.1007/s00401-009-0581-5

Neumann, M., Sampathu, D. M., Kwong, L. K., Truax, A. C., Micsenyi, M. C., Chou, T. T., et al. (2006). Ubiquitinated TDP-43 in frontotemporal lobar degeneration and amyotrophic lateral sclerosis. Science 314, 130-133. doi: 10. 1126/science. 1134108

Nolan, M., Scott, C., Gamarallage, M. P., Lunn, D., Carpenter, K., McDonough, E., et al. (2020). Quantitative patterns of motor cortex proteinopathy across ALS genotypes. Acta Neuropathol. Commun. 8:98. doi: 10.1186/s40478-020-00 961-2

Orozco, D., Tahirovic, S., Rentzsch, K., Schwenk, B. M., Haass, C., and Edbauer, D. (2012). Loss of fused in sarcoma (FUS) promotes pathological Tau splicing. EMBO Rep. 13, 759-764. doi: 10.1038/embor.2012.90

Peng, A. Y. T., Agrawal, I., Ho, W. Y., Yen, Y. C., Pinter, A. J., Liu, J., et al. (2020). Loss of TDP-43 in astrocytes leads to motor deficits by triggering A1-like reactive phenotype and triglial dysfunction. Proc. Natl. Acad. Sci. U.S.A. 117, 29101-29112. doi: 10.1073/pnas.200780 6117 
Piguet, O., and Kumfor, F. (2020). Frontotemporal dementias: main syndromes and underlying brain changes. Curr. Opin. Neurol. 33, 215-221. doi: 10.1097/ WCO.0000000000000792

Portz, B., Lee, B. L., and Shorter, J. (2021). FUS and TDP-43 phases in health and disease. Trends Biochem. Sci. 46, 550-563. doi: 10.1016/j.tibs.2020.12.005

Pottier, C., Ravenscroft, T. A., Sanchez-Contreras, M., and Rademakers, R. (2016). Genetics of FTLD: overview and what else we can expect from genetic studies. J. Neurochem. 138(Suppl. 1), 32-53. doi: 10.1111/jnc.13622

Raju, C. S., Goritz, C., Nord, Y., Hermanson, O., Lopez-Iglesias, C., Visa, N., et al. (2008). In cultured oligodendrocytes the A/B-type hnRNP CBF-A accompanies MBP mRNA bound to mRNA trafficking sequences. Mol. Biol. Cell 19, 3008 3019. doi: 10.1091/mbc.E07-10-1083

Ratti, A., and Buratti, E. (2016). Physiological functions and pathobiology of TDP43 and FUS/TLS proteins. J. Neurochem. 138(Suppl. 1), 95-111. doi: 10.1111/ jnc. 13625

Rohan, Z., Matej, R., Rusina, R., and Kovacs, G. G. (2014). Oligodendroglial response in the spinal cord in TDP-43 proteinopathy with motor neuron involvement. Neurodegener. Dis. 14, 117-124. doi: 10.1159/000362929

Romano, M., Buratti, E., Romano, G., Klima, R., Del Bel Belluz, L., Stuani, C., et al. (2014). Evolutionarily conserved heterogeneous nuclear ribonucleoprotein (hnRNP) A/B proteins functionally interact with human and Drosophila TAR DNA-binding protein 43 (TDP-43). J. Biol. Chem. 289, 7121-7130. doi: 10.1074/ jbc.M114.548859

Saez-Atienzar, S., Bandres-Ciga, S., Langston, R. G., Kim, J. J., Choi, S. W., Reynolds, R. H., et al. (2021). Genetic analysis of amyotrophic lateral sclerosis identifies contributing pathways and cell types. Sci. Adv. 7:eabd9036. doi: 10 . 1126/sciadv.abd9036

Schirmer, L., Mobius, W., Zhao, C., Cruz-Herranz, A., Ben Haim, L., Cordano, C., et al. (2018). Oligodendrocyte-encoded Kir4.1 function is required for axonal integrity. Elife 7:e36428. doi: 10.7554/eLife.36428

Seiberlich, V., Bauer, N. G., Schwarz, L., Ffrench-Constant, C., Goldbaum, O., and Richter-Landsberg, C. (2015). Downregulation of the microtubule associated protein tau impairs process outgrowth and myelin basic protein mRNA transport in oligodendrocytes. Glia 63, 1621-1635. doi: 10.1002/glia.22832

Shaker, M. R., Pietrogrande, G., Martin, S., Lee, J. H., Sun, W., and Wolvetang, E. J. (2021). Rapid and efficient generation of myelinating human oligodendrocytes in organoids. Front. Cell. Neurosci. 15:631548. doi: 10.3389/fncel.2021.631548

Sproviero, D., La Salvia, S., Giannini, M., Crippa, V., Gagliardi, S., Bernuzzi, S., et al. (2018). Pathological proteins are transported by extracellular vesicles of sporadic amyotrophic lateral sclerosis patients. Front. Neurosci. 12:487. doi: 10.3389/fnins.2018.00487

Stone, S., Wu, S., Nave, K. A., and Lin, W. (2020). The UPR preserves mature oligodendrocyte viability and function in adults by regulating autophagy of PLP. JCI Insight 5:e132364. doi: 10.1172/jci.insight.132364

Takebayashi, H., and Ikenaka, K. (2015). Oligodendrocyte generation during mouse development. Glia 63, 1350-1356. doi: 10.1002/glia.22863

Tepavcevic, V. (2021). Oligodendroglial energy metabolism and (re)myelination. Life (Basel) 11:238. doi: 10.3390/life11030238

Thelen, M. P., and Kye, M. J. (2019). The role of RNA binding proteins for local mRNA translation: implications in neurological disorders. Front. Mol. Biosci. 6:161. doi: 10.3389/fmolb.2019.00161

Torvund-Jensen, J., Steengaard, J., Reimer, L., Fihl, L. B., and Laursen, L. S. (2014). Transport and translation of MBP mRNA is regulated differently by distinct hnRNP proteins. J. Cell Sci. 127(Pt 7), 1550-1564. doi: 10.1242/jcs.140855
Uchida, T., Tamaki, Y., Ayaki, T., Shodai, A., Kaji, S., Morimura, T., et al. (2016). CUL2-mediated clearance of misfolded TDP-43 is paradoxically affected by VHL in oligodendrocytes in ALS. Sci. Rep. 6:19118. doi: 10.1038/srep1 9118

Ueno, Y., Zhang, N., Miyamoto, N., Tanaka, R., Hattori, N., and Urabe, T. (2009). Edaravone attenuates white matter lesions through endothelial protection in a rat chronic hypoperfusion model. Neuroscience 162, 317-327. doi: 10.1016/j. neuroscience.2009.04.065

van Rheenen, W., Shatunov, A., Dekker, A. M., McLaughlin, R. L., Diekstra, F. P., Pulit, S. L., et al. (2016). Genome-wide association analyses identify new risk variants and the genetic architecture of amyotrophic lateral sclerosis. Nat. Genet. 48, 1043-1048. doi: 10.1038/ng.3622

Vance, C., Rogelj, B., Hortobagyi, T., De Vos, K. J., Nishimura, A. L., Sreedharan, J., et al. (2009). Mutations in FUS, an RNA processing protein, cause familial amyotrophic lateral sclerosis type 6. Science 323, 1208-1211. doi: 10.1126/ science. 1165942

Wang, J., Ho, W. Y., Lim, K., Feng, J., Tucker-Kellogg, G., Nave, K. A., et al. (2018). Cell-autonomous requirement of TDP-43, an ALS/FTD signature protein, for oligodendrocyte survival and myelination. Proc. Natl. Acad. Sci. U.S.A. 115, E10941-E10950. doi: 10.1073/pnas.1809821115

Wang, Y., Lacroix, G., Haines, J., Doukhanine, E., Almazan, G., and Richard, S. (2010). The QKI-6 RNA binding protein localizes with the MBP mRNAs in stress granules of glial cells. PLoS One 5:e12824. doi: 10.1371/journal.pone. 0012824

White, M. A., Kim, E., Duffy, A., Adalbert, R., Phillips, B. U., Peters, O. M., et al. (2018). TDP-43 gains function due to perturbed autoregulation in a Tardbp knock-in mouse model of ALS-FTD. Nat. Neurosci. 21, 552-563. doi: 10.1038/ s41593-018-0113-5

Wu, R., Zhou, D., Shen, X., Chen, F., Liu, F., and Gu, J. (2021). Phosphorylation of Trans-active response DNA binding protein-of $43 \mathrm{kDa}$ promotes its cytoplasmic aggregation and modulates its function in tau mRNA stability and exon 10 alternative splicing. J. Neurochem. 158, 766-778. doi: 10.1111/jnc. 15450

Zhang, Y., Chen, K., Sloan, S. A., Bennett, M. L., Scholze, A. R., O’Keeffe, S., et al. (2014). An RNA-sequencing transcriptome and splicing database of glia, neurons, and vascular cells of the cerebral cortex. J. Neurosci. 34, 11929-11947. doi: 10.1523/JNEUROSCI.1860-14.2014

Conflict of Interest: The authors declare that the research was conducted in the absence of any commercial or financial relationships that could be construed as a potential conflict of interest.

Publisher's Note: All claims expressed in this article are solely those of the authors and do not necessarily represent those of their affiliated organizations, or those of the publisher, the editors and the reviewers. Any product that may be evaluated in this article, or claim that may be made by its manufacturer, is not guaranteed or endorsed by the publisher.

Copyright (c) 2021 Valori and Neumann. This is an open-access article distributed under the terms of the Creative Commons Attribution License (CC BY). The use, distribution or reproduction in other forums is permitted, provided the original author(s) and the copyright owner(s) are credited and that the original publication in this journal is cited, in accordance with accepted academic practice. No use, distribution or reproduction is permitted which does not comply with these terms. 